Conclusions Our data showed that Hsp90 promotes MSCs migration, elevate the expression of MMP-2, MMP-9, CXCR4 and VCAM1. PI3K/Akt and ERK signalling pathways mediates these effects. Hsp90 is a candidate drug for enhancement of MSCs migration.

\section{e0205 MICRORNA REGULATION OF CARDIOMYOCYTE LINEAGES FROM BONE MARROWDERIVED MESENCHYMAL STEM CELLS}

doi:10.1136/hrt.2010.208967.205

Wu Rongrong, Mo Xiaozhou, Hu Xinyang, Wang Yaping, Xiang Chunsheng, Wang Jian-an. Second Affiliated Hospital, Zhejiang University College of Medicine, Hangzhou, China

Objective ischaemic heart disease is the leading cause of morbidity and mortality all over the world. Cardiomyocytes from bone marrow-derived mesenchymal stem cells (MSCs) offer great potential for repairment of the infracted heart. However, this approach has been limited by inefficient differentiation of MSCs into cardiomyocytes. To overcome such a problem, the underlying regulation mechanisms for cardiac differentiation should be elucidated. MicroRNAs (miRNAs) are small noncoding RNAs of $\sim 23$ nucleotides that control post-transcriptional gene expression. Recently, miRNA has been widely shown to regulate key cellular events such as cell proliferation, cell differentiation. The purpose of this study is to determine the role of miRNAs during cardiac differentiation from MSCs.

Method Firstly, we established a model of cardiac differentiation from rat bone marrow-derived MSCs using $10 \mu \mathrm{M}$ 5-Aza, and performed a global miRNA analysis using EXIOON miRNA array to identify characteristic miRNA at different stage of differentiation. After being validated by real-time qRT-PCR and target gene prediction, several miRNAs such as miRNA-145 were further chosen to reveal its function during cardiac differentiation.

Results miRNA profiling revealed that miR-145 expression increased during cardiac differentiation, especially at 12 days of treatment (2.25-fold change vs untreated MSCs). Compared to other tissues such as liver, brain, kidney, miRNA-145 expression is highest in rat heart tissue. Gain-of-function methods using pre-miR-145 showed the enchancement of cardiac differentiation, confirmed by immunocytochemical staining with cardiac-specific myosin heavy chain antibody.

Conclusion miR-145 may be a critical regulator for cardiomyocyte lineage in our 5-Aza-induced differentiation system.

\section{e0206 BONE MARROW MESENCHYMAL STEM CELL MIGRATION DEPENDS IN PART ON KV21 CHANNEL ACTIVITY}

doi:10.1136/hrt.2010.208967.206

Hu Xinyang, Yu Shanping, Wang Jian-an, Wei Ling. Second Affiliated Hospital, Zhejiang University College of Medicine, Hangzhou, China

Objective The present study was designed to examine 1 . whether hypoxic preconditioning (HP) increases the migration potential of mesenchymal stem cells (MSC) and 2. the mechanistic basis for this effect.

Methods MSCs derived from green fluorescent protein (GFP)transgenic mice were cultured under either normoxia (N-MSC) or hypoxia $\left(0.5 \% \mathrm{O}_{2}\right)$ (HP-MSC) for $24 \mathrm{~h}$. Wound healing and transwell assays were performed to assess cell migration in vitro. Potassium channel expression and FAK phosphorylation were analysed by western blot analysis, whole cell patch clamp recording was used to measure delayed rectifier $\mathrm{K}^{+}$currents. Interactions between the Kv2.1 potassium channel and FAK were analysed via immunoprecipitation. N-MSC or HP-MSC were intravenously injected via tail vein in myocardial rat, and migration of MSC was assessed in vivo.

Results Both wound healing and transwell assays showed that, compared with controls, shRNA-mediated knock-down of the Kv2.1 potassium channel significantly reduced the ability of $\mathrm{N}$ MSC to migrate. While exposure to hypoxia enhanced MSC migration both in vitro and in vivo, this effect was inhibited in presence of either tetra-ethylammonium (TEA) or elevated extracellular potassium. The potential effects of hypoxia exposure on MSC migration were significantly reduced by Kv2.1 knockdown. Hypoxic pre-conditioning significantly increased the expression of Kv2.1. Similarly, compared to normoxic controls, delayed rectifier $\mathrm{K}^{+}$currents in HP-MSC were much greater. Lastly, HP-mediated increases in phosphorylation of FAK ${ }^{576 / 577}$ and FAK $^{397}$ were neutralised by either addition of TEA or elevation of extra-cellular potassium levels.

Conclusion These findings demonstrate that KV2.1 plays an important role in the regulation of MSC migration. HP enhances the ability of MSC to migrate both in vitro and in vivo, and this effect may be mediated, at least in part, through activation of Kv2.1 potassium channels and the FAK pathway.

\section{e0207 RB1 REVERSES $\mathbf{H}_{\mathbf{2}} \mathbf{0}_{\mathbf{2}}$ INDUCED SENESCENCE IN HUMAN UMBILICAL ENDOTHELIAL CELLS VIA MODULATING ENOS PATHWAY}

doi:10.1136/hrt.2010.208967.207

Liu Dinghui, Qian Xiaoxian. The Third Affiliated Hospital of Sun Yat-sen University, Guangzhou, China

Objective Cellular senescence of endothelial cells has been proposed for its involvement in endothelial dysfunction and atherogenesis. This study investigates the effects of ginsenoside Rb1, a major constituent of ginseng, on $\mathrm{H}_{2} \mathrm{O}_{2}$-induced endothelial senescence and molecular changes in primary human umbilical vein endothelial cells.

Methods Prematurely senescent human umbilical vein endothelial cells (HUVECs) were induced by treatment with $\mathrm{H}_{2} \mathrm{O}_{2}$ as judged by senescence-associated $\beta$-galactosidase assay (SA- $\beta$-gal), cell morphological appearance, and plasminogen activator inhibitor-1 (PAI-1. expression. Total nitric oxide (NO) production was measured using Griess reaction. Endothelial NOS (eNOS), PAI RNA expressions were analysed by real time PCR. Total eNOs, pS1177 eNOS and pT495 eNOS protein expressions were analysed by westernblotting.

Results Treatment with $40 \sim 100 \mu \mathrm{M} \mathrm{H}_{2} \mathrm{O}_{2}$ caused $26.8 \sim 63.8 \%$ of the cells to be SA- $\beta$-gal positive. Pretreated with $\mathrm{Rb} 1$ markedly inhibited SA- $\beta$-gal activity dose-dependently. Also, Rb1 can reduce the expression of PAI which was increased in the $\mathrm{H}_{2} \mathrm{O}_{2}$ treated group. In $\mathrm{H}_{2} \mathrm{O}_{2}$ treated groups, eNOS mRNA expression decreased, while $\mathrm{Rb} 1$ can effectively restore its mRNA expression. eNOS activity of HUVECs was inhibited by decreasing eNOS phosphorylation at Ser-1177 and increasing eNOS phosphorylation at Thr-495 in $\mathrm{H}_{2} \mathrm{O}_{2}$ treated groups. While in $\mathrm{Rb} 1$ pretreated groups, the both exhibited opposite changes. Consistent with these findings, $\mathrm{Rb} 1$ does in fact increase NO levels. All the inhibitory effects of Rb1 on senescence were completely obliterated by L-NAME, the NOS inhibitor.

Conclusion Rb1 can effectively protect HUVEC from senescence through modulating the expression of eNOS. 